\title{
Gingival esthetic of upper maxillary anterior teeth - A review of literature
}

\author{
Ahmed Al ahmari ${ }^{1}$, Badr Bamusa ${ }^{2}$, Waleed Bakhadher ${ }^{*}$, Sanaa shafshak ${ }^{4}$ and Mohmmed Ayed \\ ${ }^{1}$ Department of Periodontics, Director of Khamis Mushait Specialty Dental Center, Ministry of Health, Saudi Arabia \\ ${ }^{2}$ Department of Periodontology, Riyadh Elm University, Riyadh, Saudi Arabia \\ ${ }^{3}$ Associate Consultant in Orthodontics, Makkah, Saudi Arabia \\ ${ }^{4}$ Professor of Periodontology, Riyadh Elm University, Riyadh, Saudi Arabia \\ ${ }^{5}$ Department of Periodontology, Aseer Dental Adminstration, Saudi Arabia
}

\begin{abstract}
Gingival tissue was considered as one of important components of esthetic smile. Good prosthesis surrounded by unaesthetic gingival tissues has negatively impact on final smile. Gingival health is among the first fundamental esthetic objectives during treatment planning, it is also essential to consider gingival morphology and contour The literature presented differing information on where the gingival zenith position (GZP) is located from the vertical bisected midline (VBM) axis of each individual maxillary anterior tooth and where it should be placed
\end{abstract}

\section{Introduction}

Understanding the dentogingival interface will allow clinicians to achieve a more satisfactory esthetic outcome during interdisciplinary diagnosis and treatment [1]. Adjunctive therapies, including periodontal plastic surgery, are recommended to optimize gingival contours for restorative treatment in the presence of severe gingival deformity [2]. The gingival zenith (GZ) is defined as the most apical point of the marginal gingival scallop, however, its quantitative orientation in the apico-coronal (AC) and mesio-distal (MD) directions has not been reported [1]. The GZ has always been an important component of a beautiful smile. Generally, crooked and discolored teeth result in a distorted GZ position. Gingival asymmetry can lead to visual stress and imbalance. The disfigurement associated with rotated (crooked) anterior teeth causes physical, emotional, and social problems [3].

\section{The gingival esthetic}

The gingival tissues form the soft-tissue framework around every tooth. Healthy free gingiva extends from the free gingival margin coronally to the gingival groove apically, and it is keratinized and has a coral pink surface. The attached gingiva extends from the free gingival groove coronally to the mucogingival junction and has a coral pink color and firm texture. The attached gingiva is keratinized and can be highly stippled or smooth. The alveolar mucosa is apical to the mucogingival junction, with a loose and darker appearance [4]. The primary aim of periodontal therapy is to maintain the health as well as esthetic appearance of the gingiva. An irregular arrangement of the gingival tissues, despite being healthy, may diminish the outcome of all other treatment, resulting in a less than optimal esthetic outcome. In any esthetic treatment planning, the assessment of gingival tissues is essential and it may become desirable to establish a certain balance and symmetry of the gingival tissues to achieve a healthy and predictable final esthetic result. Assessment of gingival esthetics is of critical importance in patients with high lip lines, where the gingival margins are clearly visible, as well as for patients with high esthetic demands [5]. Treatment methods for developing gingival harmony may involve periodontal plastic surgery or orthodontics. Periodontal surgery may involve additive or resection gingival techniques, and orthodontics may involve extrusion or intrusion techniques. The various procedures and the treatment provided will ultimately depend on the underlying concern. Excess gingiva can be corrected using resection periodontal surgery techniques, such as the gingivectomy or crown lengthening surgery involving osseous recontouring of one or more teeth. Gingival recession can be corrected using additive periodontal surgical techniques, such as tissue grafts or guided tissue regeneration, or nonsurgically using orthodontic extrusion techniques [6-9]. Gingival esthetics relies on achieving a balance of gingival levels from the central incisors to the canines. The gingival architecture of the central incisor teeth should mirror one another. The gingival contour of the lateral incisors should lie more coronal to the central incisors and canines and be bilaterally symmetrical. This ideal scenario represents the Class 1 gingival height. Variations in the positioning of the gingival margin of the lateral incisors can occur; in Class 2 gingival height, the gingival contour of the lateral incisors lies apical to that of the central incisors and canines. The canines, in turn, should ideally have the level of the free gingival margin at the same height as the central incisor teeth and matching one another [10].

When evaluating the free gingival margin, the maxillary and the mandibular lateral incisors should display a semicircular shape. The

${ }^{\star}$ Correspondence to: Waleed Bakhadher, Associate Consultant in Orthodontics, Makkah, Saudi Arabia, E-mail: waleed505@live.com

Key words: gingival zenith, interdental papilla, maxillary anterior teeth, smile analysis

Received: February 25, 2019; Accepted: March 15, 2019; Published: March 19, 2019 
maxillary central incisors and canines display a more elliptical gingival shape, with the most apical point of the gingival outline positioned distal to the longitudinal axis of the tooth; this is termed the gingival zenith. The gingival zenith of the maxillary and the mandibular lateral incisors is usually centered along the tooth axis. The position of the gingival zenith around individual teeth is primarily related to tooth morphology and corresponds to the area of maximum convexity of the tooth at the free gingival margin [10]. In a healthy periodontium, the interdental papilla should fill the space below the contact area of adjacent teeth. After periodontal disease, tooth loss, or poor restorative dentistry, the interdental papilla may be lost, resulting in what is termed "dark triangles." The presence of black triangles will result in gingival disharmony and an overall compromised esthetic outcome. The presence of the interdental papilla is primarily dependent on the existence of the teeth, the underlying periodontal support, and the gingival biotype. However the relationship of the contact points of the maxillary teeth with the alveolar crest also has a significant influence on the interdental papilla. When the distance from the contact point to the interproximal osseous crest is less than $5 \mathrm{~mm}$, there is complete papillary fill of the gingival embrasures. As this distance increases for every $1 \mathrm{~mm}$ more than $5 \mathrm{~mm}$, the chances of achieving complete fill is progressively reduced by $50 \%$. The clinician should be aware of the negative esthetic impact that the lack of interdental papilla can have on dental esthetics, particularly in a patient with a high smile line. Numerous techniques can be used to optimize this aspect of "pink esthetics," through the use of esthetic periodontal surgery, orthodontics, or fixed prosthodontics, alone or in combination [11]. The ideal gingival architecture has been described as one that consists of knifeedged gingival margins tightly adapted to the teeth, interdental grooves, and coneshaped interdental papilla [6].

\section{The gingival zenith positions, levels and angles}

Ahmad [12], defined the gingival zenith (GZ) as the most apical aspect of the free gingival margin and located according to the long axis of the tooth. In the central incisor and canine the (GZ) is observed distally to the long axis. While, for the lateral incisor is, on the center of the tooth. Rufenacht [10], proposed that the gingival zenith position (GZP) was distally displaced on the central incisors and canines only, whereas those of the lateral incisors were coincident with the vertical bisected midline (VBM). Magne and Belser [13], suggested that the (GZP) was distal to the long axis of all the maxillary anterior teeth. Goodlin [14], measured the gingival zenith position (GZP) the most apical point of the free gingival margin of the periodontium, and the GZL of the lateral incisor relative to the central incisors and canine teeth can significantly influence the esthetic appearance of a smile, His results described the GZP for central incisors at the distal third, laterals at the $\mathrm{VBM}$, and canines ranging from the anterior third to the distal third of the VBM. Mattos and Santana [15], evaluated the spatial displacement of GZ in the maxillary anterior dentition, found that GZ is not universally displaced towards distal aspect and the frequency and magnitude of distal displacement is found to be larger in central incisor, than in lateral which in turn is larger than canine. The gingival zenith (GZ) has always been an important component of a beautiful smile which is defined as the most apical point of the marginal gingival scallop, however its quantitative orientation in the apico-coronal (AC) and mesio-distal (MD) directions has not been reported [1]. Chu et al. [1], explained and demonstrated the gingival zenith position (GZP) and the gingival zenith level (GZL) in details through the clinical study of 20 healthy patients by using study casts and digital caliper ; The gingival zenith positions (GZP) represented to the highest points of the free gingival margins was measured from the vertical bisected midlines along the long axis of the maxillary anterior teeth in mediallateral relations They measured GZP from VBM along the long axis of anterior teeth and GZL of the lateral incisors in an apical - coronal direction relative to the line joining the tangent of the GZP of adjacent central and canine; their study demonstrated that all central incisors displayed a distal GZP from the VBM, with a mean average of $1 \mathrm{~mm}$. Lateral incisors showed a deviation of the gingival zenith with a mean average of $0.4 \mathrm{~mm}$ distally and canines on the center with mean average of $0 \mathrm{~mm}$. Zagar et al. [16], evaluated specific distal displacement of the gingival zenith in the maxillary anterior dentition in young adults. Their results revealed that the gingival zenith was distally displaced between 0.45 and $1.01 \mathrm{~mm}$ in $90 \%, 85 \%$ and $60 \%$ of the central incisors, lateral incisors and canines. Most of the previous studies on the (GZP) found difficulty to determine the exact placement in relation to the long axis of each tooth of the six maxillary anterior teeth. The appropriate placement of the gingival zenith is critical, as it helps to determine the desired axial inclination of the tooth by maneuvering the line angle of the tooth vertical axis. Subsequently, knowing the GZP of each maxillary anterior tooth from the (VBM). While, the (GZL) of the lateral incisors can help and facilitate a reference point during esthetic periodontal plastic surgery procedures [1]. Bhatsange [17], analyzed the gingival zenith position (GZP) of maxillary central incisors in relation to vertically bisected midline by means of dental casts and digital caliper in different facial forms. The results according to the different face types were; the mean distance of GZP in relation to vertically bisected midline of maxillary incisors was $1.06 \mathrm{~mm}$ in oval, $1.12 \mathrm{~mm}$ in square, $1.04 \mathrm{~mm}$ in square tapered and $1.04 \mathrm{~mm}$ tapered. Comparison between the groups was statistically significant. The contralateral comparisons revealed no statistical difference between maxillary left and right central incisor thus emphasizing bilateral symmetry.

The gingival aesthetic line (GAL) was defined as a line joining the tangent of the gingival margin zeniths from the central incisor and canine. The GAL angle was determined at the intersection of the GAL and the maxillary midline between the two central incisors (extending through the tip of interdental papilla) [12]. Rufenacht [10], concluded that in Class 1 occlusion, the ideal GZL should be where the gingival contours of the central incisors and canines are at the same level and the lateral incisor positioned slightly more coronal. While, in Class 2 division 2 malocclusions; the GZL of the lateral incisors are more apical compared with that of the central incisors and canines, as the laterals tend to overlap the distal aspects of the central incisors. The subsequent tooth and root positions of the lateral incisors within the dental arch affect the gingival contours. Ahmad [12], determined the GAL angle at the intersection of the gingival aesthetic line (GAL) and maxillary midline between the two central incisors (extending through the tip of interdental papilla)., his recommendation was not specified as a quantified values and he suggested when the gingival zenith of the canine higher than that of the central incisor; the GAL angle will be in range of values, from $45^{\circ}$ to $90^{\circ}$. Charruel et al. [18], quantified some clinical parameters as lateral incisors distance (LID) from the gingival line (GL), and the gingival line angles (GLA) to evaluate the gingival line asymmetry between the right and left of the six maxillary anterior teeth. Their results revealed that the gingival margin of lateral incisors located on (GL) exactly in $15 \%$, apical to (GL) in $3.9 \%$, coronal to (GL) from (0 to $1 \mathrm{~mm}$ ) in $53.9 \%$ and coronal to (GL) more than $1 \mathrm{~mm}$ in $27.2 \%$. The gingival asymmetry showed the right side higher than the left side; when the left (GLA) were significantly greater than the right one, and that asymmetry was quantified by the absolute value of the left -right difference $4.1^{\circ}$. anterior teeth, their study revealed that gingival 
zenith of maxillary canine is apical to the zenith of central incisors because (GLA) measurements were $<90^{\circ}$ and the gingival zenith level of lateral incisor is frequently coronal to the gingival line. With regards to these parameters; no differences between genders but showed gingival asymmetry of gingival line angle with right sides higher than left sides. The subtle asymmetry quantified of left - right difference at $1.7 \pm 4.4^{0}$.

Pini et al. [19], evaluated the width/length ratio and the (GZL), by means of dental casts and digital caliper in patients with missing maxillary lateral incisors after treatment with implant placement and with tooth re- contouring and control group. Results concluded that group treated with canine re-contouring showed the greatest differences in values with regard to the median ratio of GZL $0.58 \mathrm{~mm}$ ; (0.5 right and 0.48 left), but the GZL for the implants replaced the bilateral agenesis was $0.95 \mathrm{~mm}$; (0.95 right and 0.98 left) , and $0.98 \mathrm{~mm}$ $;(0.98$ right and 0.8 left $)$ in the control group. The GZL for the right and left sides during the smile were not statistically different.

The samples in the previous studies were selected under specified criteria when were not having any signs of dental and periodontal disease, no missing in the maxillary anterior teeth, and excluded who have; evidence or history of dental alteration as restorative treatment, trauma, wearing and abrasion. Gingival enlargement, inflammation, altered passive eruption, attachment loss, gingival recession and history of periodontal surgery, marked spacing and severe crowding were also excluded [1,18,20]. Pawar et al. [20], quantified some clinical parameters useful as esthetic guidelines when gingival contour was modified and compared left and right sides of six maxillary

\section{The width and height of normal clinical crown}

Shaw [21], observed the influence of the social attractiveness of 800 young adults and dento-facial appearance. The attractiveness recorded on a visual analogue scale. For the majority of characteristics examined, the normal incisor relationship gained the most favorable ratings for perceived friendliness, social class, popularity and intelligence. They assessed the attractiveness of standardized alterations in incisor proportion of three individual groups (24 dentists, 24 medical students, and 179 patients). The results showed that the width/length ratio for medical students and patients $75-85 \%, 75-80 \%$ for dentists. These results was agreed to Brisman [22]. who found the most attractive proportion $80 \%$ for patients and $66 \%$ for dentists.

Sterrett et al. [23], analyzed the width, length, and the width/ length ratio of the central, lateral and canine of the maxillary anterior teeth in 71 Caucasian subjects. The study was based on stone casts measurements using digital calipers. The results revealed that the males have wider teeth than females $(\mathrm{mm})(\mathrm{CI}: 8.59$ versus 8.06 . LI: 6.59 versus 6.13. CA: 7.64 versus 7.15 ), and longer (mm) (CI: 10.19 versus 9.39. LI: 8.70 versus 7.79 . CA; 10.06 versus 8.89 ). According to the width/length ratio between both gender (males/females); there was no significant differences in ratio between CI 0.85 versus 0.86 and LI 0.76 versus 0.79 . The Canine ratio significantly greater in females 0.81 than in males 0.77 . Different studies have been suggested the width to height ratios in central incisors 0.76 on clinical crowns of human teeth [24], and 0.78 [13].

Cooper et al. [25], evaluated the width-to-height ratio of maxillary central incisors through cross-sectional study; photographs from 96 females that was voluntary and unstrained were analyzed; (three images were created; central incisors with normal form, tooth wear and delayed apical migration, the images in each set were ranked in order of most to least attractive by 32 dentists, 32 technicians and 32 patients). They suggested the normal and the most attractive widthto-height ratio for the central incisors is $82 \%$. They concluded that the aesthetic perception of the central incisor ratio varies between dentists, dental technicians and patients; and is also are unknown. The individual variability in patient response should be taken into account during treatment planning. Understanding the aesthetic perception of altered central incisor ratio is important for the treatment planning, and the knowledge of the ideal width-to-height ratio can facilitate decision making and provide guidance for achieving an aesthetic results. Modification of the gingival contour solve the tooth proportion discrepancies [5,913]. Marginal tissue recession around teeth and/ or dental implants is not only of concern to periodontal $[23,26]$ or periimplant health but also to esthetic outcomes [27,28]. As such, soft tissue augmentation, with autogenic, allogeneic, or synthetic grafts, is widely used to increase tissue volume, amount of attached KG, and/ or improve esthetic outcomes. In recent years, the main focus of periodontal plastic or reconstructive surgery was on the management of exposed root surfaces or implantabutment interfaces with the aims of increasing the amount of $\mathrm{KG}$ and attached gingival (AG), achieving root or implant-abutment coverage, and enhancing esthetic outcomes [29].

\section{The interdental papilla}

The interdental papilla is the portion of the gingiva between two adjacent teeth. The interdental papilla was at one time considered gingival tissue, roughly pyramidal in shape, with the sole function of deflecting food debris. In reality the function of the papilla is more complex, and it is a biologic barrier to protect periodontal ligament, cementum and alveolar bone from the oral environment [30]. The presence or absence of the interdental papilla depends upon the distance between the contact point to the crest of bone. When the distance from the contact point to the bone was $5 \mathrm{~mm}$ or less, the papilla was present almost $100 \%$ of the time. With a distance of $6 \mathrm{~mm}$, the papilla was present $56 \%$ of the time, and when the distance measured $7 \mathrm{~mm}$ or more, the papilla was present $27 \%$ of the time or less [8].

Loss of the interproximal dental papillae may cause functional, phonetic and aesthetic problems. Complete and predictable restoration of lost interdental papillae remains one of the biggest challenges in periodontal reconstructive surgery. It is therefore imperative to maintain the integrity of the papilla during restorative and surgical procedures [31]. Cardaropoli et al. [32], proposed new clinical index to classify the papilla presence in relation to cementoenamel junction, (CEJ), papilla position and adjacent teeth: (i) Papilla Presence Index score 1 (PPI 1), the papilla is completely present, fill the embrasure completely, and on the same level of the adjacent papilla. (ii) Papilla Presence Index score 2 (PPI 2), the papilla is incompletely present, apically to the contact point, not on the same level of the adjacent papilla, but the interproximal cementoenamel junction (iCEJ) is invisible. However, Both PPI 1 and PPI 2 could be affected by the recession buccally and subclassified as PPI 1r and PPI 2r. (iii) Papilla Presence Index score 3 (PPI 3), the papilla receded apically, and interproximal cementoenamel junction (iCEJ) is seen. (iv) Papilla Presence Index score 4 (PPI 4), the recession is combined to interproximal cementoenamel junction (iCEJ) and buccal. Most of the previous studies have concentrated on the visual location and amount of midfacial tooth and gingival display related to the upper lip. Tooth form or shape is categorized frequently as ovoid, triangular or square. Empirical reports indicate that tooth shape may affect the shape of the periodontium [33-35].

Teeth with tapered, triangular-shaped crowns have been described as having a thin gingival „,, biotype”, with highly scalloped gingival 
margins, thin alveolar bone and small proximal contacts located near their incisal edges. Square shaped crowns, on the contrary, usually have a thick periodontal ,,,biotype” with longer and more apically positioned contacts. As a result, square-shaped teeth tend to have a lower risk of gingival recession and require less tissue to fill the interproximal space [36].

Cho et al. [37], found that the interradicular distance and the distance between the contact point and the alveolar crest have independent and combined effects on the presence or absence of the interdental papilla. Based on this information, clinicians are able to influence and maintain papilla development more effectively, but they must rely on the alveolar crest as a reference point. Martegani et al. [38], examined the distance from the base of the contact area to the crest of bone in 288 sites, and determined that at $5 \mathrm{~mm}, 6 \mathrm{~mm}$, and $7 \mathrm{~mm}$, the papilla was present $98 \%, 56 \%$, and $27 \%$ of the time, respectively. Two hundred forty interdental papilla sites in 20 healthy patients were investigated. Interdental papilla heights of maxillary anterior teeth were measured from the gingival zenith, along with clinical crown lengths. Percentages of papilla height to crown length were computed and defined as papilla proportion, mesial papilla proportion (MPP), and distal papilla proportion (DPP). Mean interdental papilla heights of maxillary anterior teeth were $4 \mathrm{~mm}$ mesially, and $4.1 \mathrm{~mm}$ distally. No significant differences were found between MPP and DPP for maxillary incisors $(\mathrm{P} \geq 0.5)$. Canines demonstrated a trend toward increased distal papilla heights. Papilla proportions were approximately $40 \%$ for all tooth groups [39]. Two basic types of gingival architecture have been described - „,"scalloped thin” and ",flat-thick”. Empirical evidence suggests areas with scalloped-thin tissue are more prone to develop recession when exposed to trauma or inflammation, while flat-thick tissues are more likely to develop deeper periodontal pockets $[40,41]$. Chow et al. [41], concluded that the Papilla height decreased $0.012 \mathrm{~mm} /$ year of age, interdental papilla display was associated significantly with different factors; age, tooth form/shape, proximal contact length, crestal bone height and interproximal gingival thickness. Other factors, such as gender, race and previous orthodontic treatment and KG/AG width had minimal impact.

Hochman et al. [42], evaluated the papilla display through maximum smiling in a (420) male and female patients, and aged from 10 to 89 years. They analyzed the interdental papilla in relation to the maxillary vermillion border, and classified the gingival smile line (high , low , and cupid's bow) and the interdental smile line (high , low, and cupid's bow), and compared the six variables according to the gender and age groups. The results revealed that the females had a higher gingival smile line $76 \%$ than males $24 \%$, and $87 \%$ of all patients with low gingival smile line had high interdental smile line, $91 \%$ displayed the interdental papilla through smiling in both high and low gingival smile groups. According to the decade of life, the papilla display decreasing when the age increasing. Spear [43], presented a clinical technique for maintaining papilla height and form following anterior tooth removal. He believed that the presence of adjacent tooth attachment and the size of the gingival embrasure formed by these teeth were responsible for papilla presence and height. Fradeani [44], described a systematic approach to esthetic analysis, evaluation, and treatment based on general principles and illustrated that the height of the interdental papillae decreased from anterior to posterior teeth. Several investigators have attempted to establish guidelines for proper papillae form to enhance denture esthetics optimize soft tissue position, improve surgical and nonsurgical techniques to treat soft tissue deformities, and to better manage interproximal spaces following tooth extraction or implant placement $[45,46]$.
It is important to respect the integrity of the papilla during dental treatment and it may be advantageous to achieve keratinization by interproximal brushing to increase the resistance of the cool area [47]. There is no universal guideline for clinicians to follow in creating greater conformity and a predictable esthetic smile, including ideal papilla heights. It may be possible to mathematically quantify certain esthetic components to establish a standard that is satisfying to both patients and clinicians, i.e., it may be feasible to quantify the heights of the interdental papillae of the maxillary anterior teeth as measured from the level of the zenith of the labial free gingival margin gingival zenith [39].

\section{Incisal embrasure morphology}

The incisal embrasures are defined as the spaces that are developed between maxillary anterior teeth as a result of the separation and edges of the teeth. These spaces help to define the individual teeth and enhance esthetics [40]. Foulger et al. [48] have shown that the lack of embrasure spaces has a significant negative impact on perceived smile esthetics, when assessed by dentists, dental technicians, and patients. One further conclusion from their study was that an incisal embrasure space arrangement where the embrasure spaces increase in size and volume distally from the midline was deemed to be the most esthetic. The embrasure spaces are approximately the shape of an inverted "V", and the size and volume of the incisal embrasures between teeth should increase in progression distally from the central incisors to the posterior dentition.

\section{Gingival embrasure space}

Gingival embrasures are defined as the embrasure cervical to the interproximal contact. If the embrasure space is not completely filled by the gingiva, it is considered open. Open gingival embrasures contribute to retention of food debris and can adversely affect the health of the periodontium. They are more common in adult patients with bone loss. Key considerations in restorative and orthodontic treatment are preserving papilla the gingival embrasures of the esthetic zone. Open embrasures are best managed with a team work involving restorative, orthodontic and periodontal parts [49].

Open gingival embrasures are visibly unaesthetic and negatively affect a person's smile. Orthodontists considered a $2 \mathrm{~mm}$ open gingival embrasure as noticeably less attractive than an ideal smile with normal gingival embrasure. Open gingival embrasures slightly greater than 3 $\mathrm{mm}$ were considered less attractive by both general dentists and the general population [50]. For better manage open gingival embrasures, the dental clinician must be aware of the underlying etiology and make an individualized treatment plan for each patient [26]. Many embrasures may be corrected with restorative procedures, but if the underlying etiology is not addressed correctly, the result may not be as esthetic as expected. Open gingival embrasures are more frequently encountered in adults undergoing orthodontic treatment $(38 \%)$ than adolescents in treatment (15\%). However, $41.9 \%$ of adolescent patients who are treated for maxillary incisor crowding have gingival embrasures. The higher prevalence in adults is related to periodontal disease and periodontal or orthognathic surgery. Gingival embrasures change over time, and once filled, embrasures may become open again [51]. Etiologic factors for open gingival embrasures include; dimensional changes of papilla during orthodontic treatment, long lasting orthodontic treatment, loss of periodontal attachment resulting in recession, loss of height of the alveolar bone relative to interproximal contact, length of embrasure area, root angulations, age, contact position, and triangular-shaped crowns. Patients may present with one or more etiologic factor; thus, 
managing each patient requires an individual assessment and treatment plan [52].

The occurrence of open gingival embrasures is found to be age related. Studies have demonstrated that patients over 20 years are more susceptible than those under 20 years. Open gingival embrasures were reported in $67 \%$ of the population over 20 years compared with $18 \%$ in the population under 20 years. This is due to the thinning of oral epithelium, a decrease in keratinization, and a reduction in papilla height as a result of the aging process. Therefore, age is a significant factor leading to wide and long embrasure spaces in adults. Embrasure and tooth morphology also is another etiologic factor. Open embrasures occurs more frequently in short narrow, long narrow, long wide, and short wide embrasure morphologies [53].

\section{Perception and smile analysis}

Beauty is constantly sought in the face, especially in the smile. One of the greatest concerns in esthetic restorations is maintenance of gingival architecture and the presence of interdental papilla to fill the interproximal spaces. Establishing harmony between the soft tissues and teeth is based on the following considerations: - The position of the free gingival margin compared to the adjacent tooth $\bullet$ The thickness of the soft tissue around the restoration • Gingival topography (flat or scalloped) • Texture and color of gingival tissue $\bullet$ Tooth shape (square, triangular, or oval) $\bullet$ Level and contour of the bone crest (which must sustain the soft tissue contour and fill the interdental space) - Threedimensional position of the implant which will contribute to the correct emergence profile and gingival contour $[54,55]$. There are some basic universally recognized principles that govern our ideas about what is considered attractive. An expressive smile is a highly salient form of nonverbal communication and possessing a smile that leaves a pleasing impression is naturally quite desirable. It is the clinician's task to balance the patient's esthetic needs with functional requirements while maintaining the individual characteristics unique to each patient. Advances in technology and biomaterials have dramatically expanded the possibilities for esthetic optimization in restorative dentistry. Too often, however, such treatment focuses exclusively on the dental area and fails to consider the overall facial composition. An evaluation of the patient's expectations and understanding of the possible therapeutic solutions is crucial before embarking upon any treatment plan [55].

Analysis of the facial features and lip movements in relation to the teeth, achieved by evaluating the facial, dentolabial, and phonetic parameters, is an indispensable first step in prosthetic rehabilitation. Consideration of the dental and gingival aspects complete the esthetic analysis, providing the treating clinician with the information needed to make the most appropriate treatment choices in each individual case. Careful evaluation of these parameters allows clinicians, by means of a practical and rational approach, to improve the quality of their work, helping them to create restorations that are harmonious not only with the smile, but also with the rest of the patient's face. To this end, the creation of a suitable esthetic checklist that includes facial, dentolabial, phonetic, tooth, and gingival analyses is invaluable for optimizing the esthetic appearance of the prosthetic rehabilitation $[5,55,56,44]$. Before focusing attention on the teeth, it is necessary to evaluate the facial composition. Frontal and lateral examination of the subject, including analysis of the position of the eyes, nose, chin, and lips allows identification of the reference points and lines that are indispensable for orienting the occlusal plane and the gingival outline in esthetic rehabilitation [55]. Dentolabial analysis focuses attention on the lower third of the face. The face and lips together form a dynamic frame for the teeth, with tooth exposure constantly changing during speaking and smiling. A systematic approach to dentolabial analysis evaluating specific parameters allows for an exhaustive assessment that will help the clinician to achieve good esthetic integration of the prosthetic rehabilitation [55,57].

\section{Declarations}

\section{Conflicts of interest}

None.

\section{Funding}

This research didn't receive any specific grants from funding agencies in the public commercial not for profit sectors.

\section{References}

1. Chu SJ, Tan JH, Stappert CF, Tarnow DP (2009a) Gingival zenith positions and levels of the maxillary anterior dentition. J Esthet Restor Dent 21: 113-120. [Crossref]

2. Blitz N (1997) Criteria for success in creating beautiful smiles. Oral Health 87: 38-42. [Crossref]

3. Singhal M, Singhal R (2012) A CAD-CAM prosthodontic option and gingival zenith position for a rotated maxillary right central incisor: An evaluation. Indian J Dent Res 23: $112-115$.

4. Sharma N, Rosenstiel SF, Fields HW, Beck FM (2012) Smile characterization by U.S. white, U.S. Asian Indian, and Indian populations. J Prosthet Dent 107: 327-335. [Crossref]

5. Rufenacht CR (2000) Principles of esthetic integration, Quintessence Publishing Company.

6. Palmer RM (1990) Free gingival graft for improvement of aesthetics. Restorative Dent 6: 8-10. [Crossref]

7. Prato GP (2000) Advances in mucogingival surgery. J Int Acad Periodontol 2: 247.

8. Tarnow DP (1992) Surgical considerations for the prosthodontic patient. Curr Opin Dent 2: 34-38. [Crossref]

9. Sato N (2000) Periodontal surgery: a clinical atlas, Quintessence.

10. Reenwell H, Fiorellini J, Giannobile W, Offenbacher S, Salkin L, et al. (2005) Oral reconstructive and corrective considerations in periodontal therapy. J Periodontol 76: $1588-600$.

11. Sarver DM, Yanosky M (2005) Principles of cosmetic dentistry in orthodontics: Part aser treatments for tooth eruption and soft tissue problems. Am J Orthod Dentofacial Orthop 127: 262-264.

12. Ahmad I (1998) Geometric considerations in anterior dental aesthetics: restorative principles. Pract Periodontics Aesthet Dent 10: 813-22.

13. Mattos CM, Santana RB (2008) A quantitative evaluation of the spatial displacement of the gingival zenith in the maxillary anterior dentition. J Periodontol 79: 1880-1885.

14. Goodlin R (2003) Cosmetic dentistry-gingival aesthetics--A critical factor in smile design. Oral Health 93: 10-28.

15. Martegani P, Silvestri M, Mascarello F, Scipioni T, Ghezzi C, et al. (2007) Morphometric study of the interproximal unit in the esthetic region to correlate anatomic variables affecting the aspect of soft tissue embrasure space. J Periodontol 78: 2260-2265.

16. Zagar M, Knezovic Zlataric D, Carek V (2010) A quantitative evaluation of the distal displacement of the gingival zenith in the maxillary anterior teeth. Coll Antropol 34: 225-228.

17. Bhatsange A (2015) A quantitative evaluation of gingival zenith position of maxillary central incisors in different facial forms. IOSR Journal of Dental and Medical Sciences.

18. Charruel S, Perez C, Foti B, Camps J, Monnet-Corti V (2008) Gingival contour assessment: clinical parameters useful for esthetic diagnosis and treatment. Journal of periodontology 79: 795-801.

19. Rufenacht CR, Berger RP (1990) Fundamentals of esthetics, Quintessence Chicago.

20. Pini NIP, De-Marchi LM, Gribel BF, Ramos AL, Furquim LZ, et al. (2012) Analysis of width/height ratio and gingival zenith in patients with bilateral agenesis of maxillary lateral incisor. Dental Press Journal of Orthodontics 17: 87-93. 
21. Shaw WC (1981) The influence of children's dentofacial appearance on their social attractiveness as judged by peers and lay adults. Am J Orthod 79: 399-415.

22. Brisman AS (1980) Esthetics: a comparison of dentists' and patients' concepts. $J$ Am Dent Assoc 100: 345-352. [Crossref]

23. Stetler KJ, Bissada NF (1987) Significance of the width of keratinized gingiva on the periodontal status of teeth with submarginal restorations. J Periodontol 58: 696-700.

24. Prichard J (1961) Gingivoplasty, gingivectomy, and osseous surgery. Journal of Periodontology 32: 275.

25. Cooper G, Tredwin C, Cooper N, Petrie A, Gill D (2012) The influence of maxillary central incisor height-to-width ratio on perceived smile aesthetics. British Dental Journal 212: 589-599.

26. Magne P, Gallucci GO, Belser UC (2003) Anatomic crown width/length ratios of unworn and worn maxillary teeth in white subjects. J Prosthet Dent 89: 453-461. [Crossref]

27. Hochman MN, Chu SJ, Tarnow DP (2012) Maxillary anterior papilla display during smiling: a clinical study of the interdental smile line. International Journal of Periodontics and Restorative Dentistry 32: 375.

28. Zigdon H, Machtei EE (2008) The dimensions of keratinized mucosa around implants affect clinical and immunological parameters. Clin Oral Implants Res 19: 387-392.

29. Fu JH, Su CY, Wang HL (2012) Esthetic soft tissue management for teeth and implants. J Evid Based Dent Pract 12: 129-142. [Crossref]

30. Caton J, Bouwsma O, Polson A, Espeland M (1989) Effects of personal oral hygiene and subgingival scaling on bleeding interdental gingiva. J Periodontol 60: 84-90.

31. Blatz MB, Hurzeler MB, Strub JR (1999) Reconstruction of the lost interproximal papilla--presentation of surgical and nonsurgical approaches. Int $J$ Periodontics Restorative Dent 19: 395-406.

32. Cardaropoli D, Re S, Corrente G (2004) The papilla presence index (PPI): A new system to assess interproximal papillary levels. Int $J$ Periodontics Restorative Dent 24: $488-492$.

33. Frush JP, Fisher RD (1958) The dynesthetic interpretation of the dentogenic concept. The Journal of Prosthetic Dentistry 8: 558-581.

34. Tjan AH, Miller GD, The JG (1984) Some esthetic factors in a smile. J Prosthet Dent 51: 24-28. [Crossref]

35. Van Der Geld P, Oosterveld P, Kuijpers-Jagtman AM (2008) Age-related changes of the dental aesthetic zone at rest and during spontaneous smiling and speech. Eur $J$ Orthod 30: 366-373.

36. Lang NP, Löe H (1972) The relationship between the width of keratinized gingiva and gingival health. J Periodontol 43: 623-627. [Crossref]

37. Cho HS, Jang HS, Kim DK, Park JC, Kim HJ, et al. (2006) The effects of interproximal distance between roots on the existence of interdental papillae according to the distance from the contact point to the alveolar crest. Journal of Periodontology 77: 1651-1657.
38. Montevecchi M, Checchi V, Piana L, Checchi L (2011) Variables affecting the gingival embrasure space in aesthetically important regions: Differences between central and lateral papillae. Open Dent J 5: 126-135.

39. Chu SJ, Tarnow DP, Tan JH, Stappert CF (2009b) Papilla proportions in the maxillary anterior dentition. Int J Periodontics Restorative Dent 29: 385-393.

40. Chang LC (2009) Effect of bone crest to contact point distance on central papilla height using embrasure morphologies. Quintessence Int 40: 507-513.

41. Chow YC, Eber RM, Tsao YP, Shotwell JL, Wang HL (2010) Factors associated with the appearance of gingival papillae. J Clin Periodontol 37: 719-727. [Crossref]

42. Kois JC (2004) Predictable single-tooth peri-implant esthetics: five diagnostic keys. Compend Contin Educ Dent 25: 895-896, 898, 900 passim. [Crossref]

43. Spear FM (1999) Maintenance of the interdental papilla following anterior tooth removal. Pract Periodontics Aesthet Dent 11: 21-28.

44. Fradeani M (2004) Esthetic analysis: a systematic approach to prosthetic treatment, Quintessence Publishing (IL).

45. Priest GF (2007) The esthetic challenge of adjacent implants. J Oral Maxillofac Surg 65: 2-12. [Crossref]

46. Pawar B, Mishra P, Banga P, Marawar PP (2011) Gingival zenith and its role in redefining esthetics: A clinical study. J Indian Soc Periodontol 15: 135-138. [Crossref]

47. Checchi L, Montevecchi M, Checchi V, Bonetti GA (2009) A modified papilla preservation technique, 22 years later. Quintessence Int 40: 303-311. [Crossref]

48. Foulger TE, Tredwin CJ, Gill DS, Moles DR (2010) The influence of varying maxillary incisal edge embrasure space and interproximal contact area dimensions on perceived smile aesthetics. Br Dent $J$ 209: E4.

49. Ravon NA, Handelsman M, Levine D (2008) Multidisciplinary care: Periodontal aspects to treatment planning the anterior esthetic zone. J Calif Dent Assoc 36: 575-584.

50. Tanaka A, Kodaira Y, Ishizaki K, Sakurai K (2008) Influence of palatal surface shape of dentures on food perception. J Oral Rehabil 35: 715-721. [Crossref]

51. Sharma AA, Park JH (2010) Esthetic considerations in interdental papilla: Remediation and regeneration. $J$ Esthet Restor Dent 22: 18-28.

52. Clark D (2009) Correction of the "black triangle": restoratively driven papilla regeneration. Dent Today 28: 154-155.

53. Mehl CJ, Harder S, Kern M, Wolfart S (2011) Patients' and dentists' perception of dental appearance. Clinical Oral Investigations 15: 193-199.

54. Ahmad I (2005) Anterior dental aesthetics: gingival perspective. Br Dent J 199: 195202. [Crossref]

55. Fradeani M (2006) Evaluation of dentolabial parameters as part of a comprehensive esthetic analysis. Eur J Esthet Dent 1: 62-69. [Crossref]

56. Chiche G, Pinault A (1994) Artistic and scientific principles applied to esthetic dentistry. Esthetics of anterior fixed prosthodontics. Quintessence Books, Chicago, pp: 13-32.

57. Olsson M, Lindhe J, Marinello CP (1993) On the relationship between crown form and clinical features of the gingiva in adolescents. J Clin Periodontol 20: 570-577. [Crossref]

Copyright: $\odot 2019 \mathrm{Al}$ ahmari A. This is an open-access article distributed under the terms of the Creative Commons Attribution License, which permits unrestricted use, distribution, and reproduction in any medium, provided the original author and source are credited. 